\title{
Danzando fakariya: los bailes uitotos como modelo de organización social en la Amazonía
}

Danser le fakariya : les bals uitoto comme modèle d'organisation sociale en Amazonie

Fakariya dancing: the Uitoto dances as a model of social organization in the Amazon

\section{Oscar Iván García}

\section{OpenEdition}

Journals

\section{Edición electrónica}

URL: http://journals.openedition.org/bifea/7805

DOI: 10.4000/bifea.7805

ISSN: 2076-5827

Editor

Institut Français d'Études Andines

Edición impresa

Fecha de publicación: 1 abril 2016

Paginación: 39-62

ISSN: 0303-7495

Referencia electrónica

Oscar Iván García, « Danzando fakariya: los bailes uitotos como modelo de organización social en la Amazonía », Bulletin de l'Institut français d'études andines [En línea], 45 (1) | 2016, Publicado el 08 abril 2016, consultado el 05 noviembre 2020. URL : http://journals.openedition.org/bifea/7805 ; DOI : https://doi.org/10.4000/bifea.7805

\section{(c)}

Les contenus du Bulletin de l'Institut français d'études andines sont mis à disposition selon les termes de la licence Creative Commons Attribution - Pas d'Utilisation Commerciale - Pas de Modification 4.0 International. 


\title{
Danzando fakariya: los bailes uitotos como modelo de organización social en la Amazonía
}

\author{
Oscar Iván García*
}

\section{Resumen}

A pesar de los importantes estudios consagrados a los ritos de la Amazonía, la comprensión de esta práctica es todavía dispersa y superficial. Actualmente existen pocos estudios comparativos que den cuenta de estas prácticas aun si los ritos amazónicos están frecuentemente ligados a intercambios simbólicos de extensión regional. Por tal razón en este documento proponemos analizar los «Bailes de Yuaki» (baile de frutas), uno de los ritos uitotos más exigentes de este grupo que es compartido por casi todas las etnias de la región del Medio Caquetá. Esta ceremonia está ligada a otros ritos y mitologías, tanto uitotos como de otros pueblos de la zona, a través de los cuales se definen las relaciones entre los grupos de este sector de la Amazonía. Con el fin de comprender la forma de concretar las relaciones entre estos pueblos en el contexto de los bailes uitotos, describiremos la manera como dos de los pueblos de este sector de la Amazonía — los mirañas y los yaguas— definen sus vínculos con los uitotos. Luego, sirviéndonos de un análisis relacional y coreográfico, dirigiremos nuestra atención a una de las partes del baile de Yuaki: las danzas de fakariya. El estudio de estos aspectos nos mostrará que a pesar de los fuertes cambios que han sufrido los pueblos de la región, estas sociedades siguen siendo determinadas por los mismos modelos simbólicos que estructuran sus ritos.

Palabras clave: contacto interétnico, danzas rituales, depredación, afinidad potencial

* Doctorante en Antropologia. École des Hautes Études en Sciences Sociales (EHESS), Laboratoire d'Anthropologie Sociale. E-mail: oigarciar@gmail.com 


\title{
Danser le fakariya : les bals uitoto comme modèle d'organisation sociale en Amazonie
}

\author{
Résumé
}

La connaissance des rites des peuples amazoniens, malgré les nombreuses recherches consacrées á ce sujet, demeure encore lacunaire. II existe peu d'études comparatives rendant compte de ces pratiques même si les rites amazoniens comportent souvent des modèles d'échange symbolique d'extension régionale. C'est pourquoi dans ce document nous avons voulu analyser les «bals de Yaukił» (bals de fruits), I'un des rites les plus exigeants et les plus diffusés dans la région du Moyen Caquetá. Ce type de cérémonie est lié à d'autres mythes et à d'autres rites, qui appartiennent tant aux Uitoto qu'à d'autres groupes de la région. Afin de mieux comprendre la façon dont les relations régionales se concrétisent dans le contexte des bals uitoto, nous décrirons d'abord la manière dont deux des peuples de la région - Miraña et Yagua — définissent leurs liens avec les Uitoto. Puis, en nous servant d'une analyse relationnelle et choréographique, nous porterons notre attention sur l'une des composantes des bals Yuaki — les danses de fakariya — afin d'analyser un troisième modèle expliquant les relations interethniques des peuples de la région. L'étude de ces aspects nous montrera que malgré les forts changements connus par les sociétés de cette région, celles-ci continuent à être déterminées par les mêmes modèles symboliques supportant leurs rites.

Mot-clés : contact interethnique, danses rituelles, prédation, affinité potentielle

\section{Fakariya dancing: the Uitoto dances as a model of social organization in the Amazon}

\begin{abstract}
Despite numerous and significant research, knowledge of Amazonian people rites is still superficial and non-cohesive. There are few comparative studies reporting such practices even if Amazonian rituals often include models of symbolic exchange at a regional level. For this reason, this article wants to examine the "Yauki balls" (balls of fruit), one of the most demanding and shared rituals in the Middle Caquetá region. This ceremony is linked to other myths and rituals from the Uitoto and other ethnic groups in the region. Therefore, to better understand how regional relations materialize in the context of the Uitoto's dances, we will describe the way in which two of the peoples of the region - Miraña and Yagua - define their ties with the Uitoto. Then, by using a relational and choreographic analysis we will approach one of the components of the Yuaki balls: Fakariya's dances. The study of these aspects will demonstrate that regardless of the strong changes experience by the peoples of the region, they remain determined by the same symbolic models that structure their rites.
\end{abstract}

Keywords: inter-ethnic contact, ritual dances, predation, potential affinity 


\section{INTRODUCCIÓN: DOS MODELOS DE ORGANIZACIÓN SOCIAL DEL NOROESTE AMAZÓNICO}

Algunos estudios etnográficos han buscado dar cuenta de la configuración social que vincula los grupos del noreste amazónico. Entre todos ellos, dos llaman la atención. El primer modelo basado en las prácticas de intercambio del pueblo yagua, estudiado por Jean-Pierre Chaumeil, se basa en un conjunto particular de intercambios mediados por la guerra. Los yaguas llevaban a cabo dos tipos de guerra. La primera de carácter regular era orientada a la búsqueda de objetos contenedores de «energía vital» (dientes humanos), robo de mujeres (intercambio) y captura de prisioneros. Tales guerras definían grupos de «enemigos próximos». En el segundo tipo de guerra, de carácter esporádico, no se tomaban ni mujeres, ni prisioneros, ni se generaba intercambio alguno. Estas definen una segunda categoría de antagonistas: los «enemigos distantes». A partir de este análisis el autor genera un modelo de organización social que comporta un eje horizontal coincidente con el río Amazonas sobre el cual se sitúan los grupos jeberos y cocamas (oeste), con quienes mantenían fluidas relaciones de intercambio, y los tikunas (oeste), con quienes los intercambios eran más limitados. En un eje relativamente perpendicular el modelo sitúa a los grupos matsés (sur) y a los uitotos (norte) con quienes no habían intercambios pero sí feroces prácticas de guerra (Chaumeil, 1985; 1994; 2006).

El segundo modelo, basado en el análisis de las categorías espaciales de la sociedad miraña es analizado por Dimitri Karadimas. El autor parte de dos nociones: «Gente de Dios» (Nìmú'e Múinaa) y Gente de los animales (İame Múinaa). La primera permite determinar un «nosotros» y la segunda para dar cuenta de ciertos grupos de alteridad. El territorio es definido en una relación de oposición centro/periferia, donde el centro es ocupado por la Gente de Dios, que en este caso vincula a todos los pueblos de lo que otros Ilaman la Gente de Centro, es decir los pueblos mirañas, boras, okainas, muinanes y uitotos, entre otros. Los confines estarían poblados por los grupos de la «Gente de los animales»: a la «izquierda» (norte) del eje fluvial de este sistema (río Caquetá) se hallan los grupos del Apaporis y del Vaupés y a la derecha (sur) los tikunas, cocamas y yaguas. Al este se hallaban los antiguos grupos tupi hoy reemplazados por los blancos, mientras que al oeste se hallaba el Ilamado «territorio de las estrellas» a través del cual se accedía a los grupos del Alto Caquetá y de los Andes (Karadimas, 2004; 2005)1.

Estos dos modelos tienen varios puntos en común: son etnocéntricos; toman como eje de proximidad un cuerpo fluvial; comportan una configuración biaxial, es decir, proponen dos ejes espaciales (relativamente perpendiculares) y describen las relaciones con grupos «locales» (eje horizontal) y de «alteridad» (eje vertical).

1 El análisis de Karadimas da cuenta del modelo de organización social miraña. No obstante, su objetivo en esta parte de su trabajo no apunta a la organización social sino a la percepción de las categorías espaciales de este grupo étnico, razón por la cual el análisis de la estructura de relaciones discutidas parece relativamente breve. 


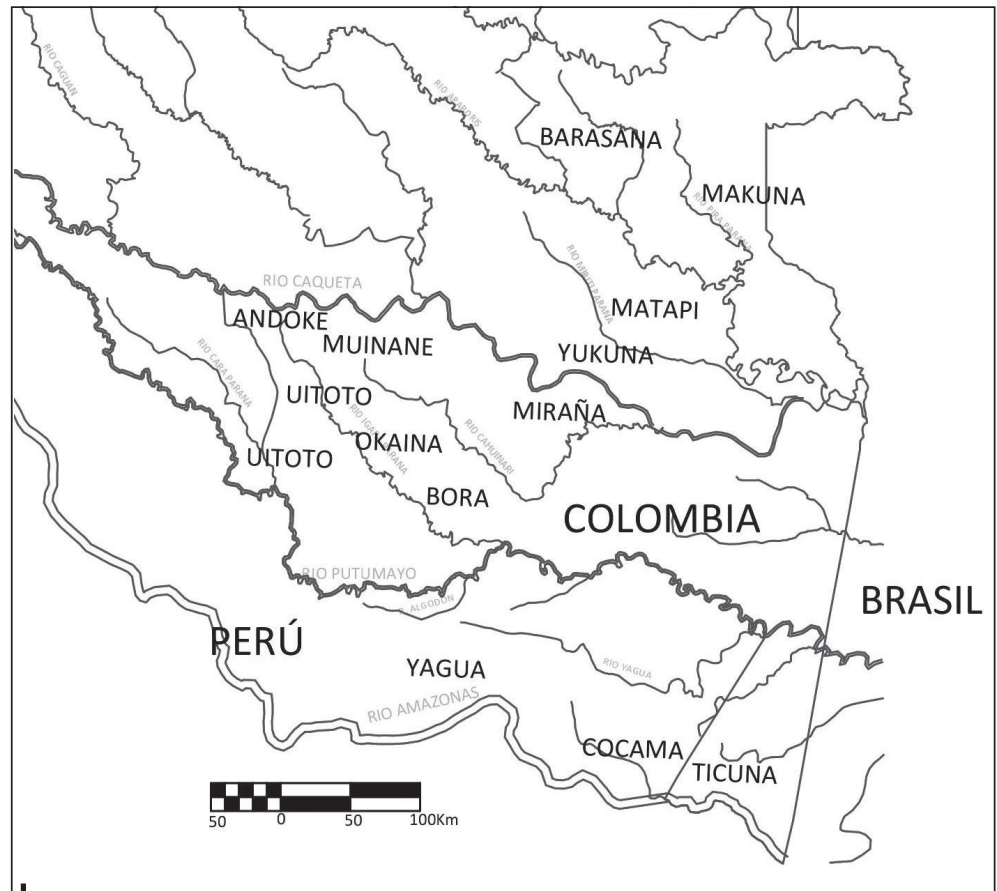

Figura 1 - Mapa de la zona de estudio

(C) Hecho a partir de http://www.bibliocad.com/biblioteca/mapacolombia_13366\#

El modelo miraña define claramente los límites del sistema, de modo que es posible reconocer su origen y su alcance pero no los puntos intermedios entre estos extremos. Tales puntos intermedios, en los términos de E. Leach (Leach et al., 1980), permiten la definición de un esquema de graduación de la alteridad entre un «nosotros» y un «otro». Al contrario, el modelo yagua tiene la virtud de mostrar tales puntos e incluso sugiere que tales niveles de gradación son siempre provisionales². Ahora bien iexisten también tales gradaciones y dinamismo

2 Este artículo no ignora la proposición enunciada por J. A. Echeverri (Franky Calvo et al., 2001: 23) quien postula un modelo que agrupa los pueblos de una parte del noroeste amazónico, particularmente los que se hallan situados dentro o cerca del interfluvio de los ríos Caquetá y Putumayo. El autor afirma que, teniendo en cuenta las «diferencias en los modelos de organización social, los sistemas ceremoniales y el desarrollo de un sistema conceptual abstracto concentrado en la Palabra [ritual]», es posible proponer un conjunto de regiones que explica el sistema regional al cual se vinculan los grupos de este sector de la Amazonía. El autor propone tres categorías: "Gente que sopla tabaco» (al norte del río Caquetá); «Gente del ambíl» (interfluvio Caquetá-Putumayo); y un tercer grupo, un poco menos claro, que incluiría «los grupos situados hacia el Amazonas por ambos lados que podría incluir grupos como los Yagua, Ticuna, etc.» (cf. Chaumeil, 2006: 53). Aun si esta propuesta no ha sido suficientemente desarrollada, es preciso decir que la misma ofrecería un modelo que podría ir más allá de la definición de «regiones discretas». En efecto, a partir de este análisis se podrían analizar los flujos de relaciones (económicas, sociales, ceremoniales, etc. ) a fin de explicar los vínculos internos de los grupos inscritos en las categorías propuestas por el autor. 
reportado para los grupos yaguas entre los grupos de la Gente de Centro? A partir del análisis del baile de Yuaki, en este documento veremos cómo los modelos de la Gente de Centro, dan cuenta de tales puntos intermedios así como de un alto grado de dinamismo que le ha permitido al sistema mantenerse vigente aún después de un prolongado periodo de contacto con la sociedad Occidental.

\section{BAILE DE YUAKt (FRUTAS)}

Los bailes uitotos son de varios tipos. Existen bailes que determinan el paso de los participantes de un estado a otro, tales como los bailes de duelo, aquellos dedicados al nacimiento o asignación de nombres rituales, etc. En general estos ritos están ligados a la evolución de las «carreras ceremoniales» de los celebrantes. Significa que corresponden a las vicisitudes que determinan la vida ritual de los Illaima (jefes). Entre las carreras ceremoniales uitotos, cuatro son las más importantes: Yadiko, Menizat, Zikiti y Yuaki, por haber sido heredadas directamente del Padre Creador Ilamado en este caso Moo Buinaima (Urbina-Rangel, 2010).

Un baile en general puede dividirse en cinco partes. La primera comienza con el cultivo de una gran chagra (campo de cultivo) destinada para la fiesta; esta parte se caracteriza por las recitaciones y discursos rituales nocturnos. La segunda parte comienza con la cosecha de la chagra de baile y la preparación de los alimentos de la fiesta. El inicio de la tercera parte está marcado por las convocatorias o invitaciones de los Fuerama o jefes Contendores³, así como por la cacería y las primeras danzas ceremoniales (danzas de fakariya). La cuarta parte del ritual corresponde al baile mismo, es decir al momento central de la actividad ceremonial; allí los grupos de cantores-danzantes se integran a la celebración como parte de un grupo invitado. La quinta parte es también el cierre de la fiesta que se lleva a cabo durante la última noche del baile. Los invitados se ocupan de entonar los cantos que fueron interpretados de manera incompleta o incorrecta así como de intervenir con otros cantos hasta terminar con los alimentos de la fiesta.

Los términos utilizados para referirse a la noción de danza en los pueblos de la Amazonía a veces desbordan la noción occidental de este fenómeno. Entre los barasana por ejemplo,

el término basa implica directamente cantos, danzas, instrumentos musicales, ornamentación corporal (plumas/pintura corporal); indirectamente basa implica la celebración de ciertas reuniones sociales, varias clases de

Así mismo, podrían comprenderse los vínculos con los grupos de regiones vecinas como los de las cuencas del Vaupés, Apaporis y Yavarí. Teniendo en cuenta que en este artículo coincidimos en analizar algunos de los criterios sugeridos por Echeverri, este texto puede ser entendido como una contribución a esta discusión a la cual comienzan a vincularse otros autores interesados en el tema.

3 La palabra fuerama deriva del término fue (primero) que puede vincularse o al sufijo nama (dueño) refiriéndose así al principal de los jefes invitados. En el español local estos jefes son llamados «Contendores» dado que en el contexto del baile estos actúan en calidad de jefes aliados tanto como de jefes de los grupos enemigos (afines). 
discursos (oratoria-canto-encantamientos, hechizos de protección), la producción de cerveza de yuca, coca, tabaco (cigarros/tabaco), etc. (HughJones, comunicación personal).

Entre los uitotos el término con el que se refieren a la acción de danzar tiene implicaciones directas e indirectas del mismo tipo; sin embargo, son varios los términos que se usan en este contexto. En primer lugar, se utiliza el término rote para hablar, de la manera más general, de la acción de cantar. No obstante este tipo de representación es notablemente diverso, tanto como los términos que los describen: un canto antifonal cantado por una mujer durante un baile ritual para acompañar a un hombre es llamado aiaide; la canción cantada por un chamán para la curación se llama j̈̈de o diuena; el tipo particular de canto interpretado durante la preparación de una baile, cantado por una persona en solitario para enseñar a los demás, se llama zomade. Los cantos entonados por los anfitriones de ciertos bailes de prestigio (Yadiko, Menizait, Zikiti,etc.) son llamados buñua; y el tipo de canto interpretado en el momento de la entrega de los intercambios de cacería por comida cultivada (tomadas) se llama fakariya. No todos los cantos son danzados, solamente algunos como los buñua, los fakariya y los cantos colectivos que son el centro de las fiestas. El término jiłyirite (danza) se usa para referirse a los movimientos acompasados con el cuerpo (brazos, pies, cabeza, etc.) puesto en escena durante una danza masculina o femenina que tiene lugar durante los cantos de fakariya o durante los cantos colectivos de un baile ritual. Sin embargo, es el término Zaite el que mejor se vincula con las acciones que se desarrollan en estos bailes; es decir, el que mejor corresponde con las implicaciones directas e indirectas de las danzas de este pueblo. Zaite se refiere a la acción de cantar y danzar al mismo tiempo. Es la acción que reúne canto y danza para generar una enunciación y para poner en marcha los dispositivos ceremoniales. Aun si un baile vincula varios tipos de ceremonias y de acciones rituales, en este documento nos concentraremos en la tercera parte de este rito. Tal decisión tiene su justificación en que durante esta parte del baile de Yuaki los zaite (cantos-danzados) ligados a los fakariya muestran mejor los intercambios y las relaciones entre grupos humanos y no humanos 4 . Los análisis que siguen se concentrarán mayormente sobre esta parte del baile.

\section{1. Los anfitriones}

Antes de analizar el grupo de los anfitriones es preciso recordar tres de las características que determinan la sociedad uitoto: la virilocalidad, la herencia patrilineal y la exogamia. La virilocalidad hace que los matrimonios residan en casa del linaje del conyugue masculino; esto, ligado a la patrilinealidad, garantiza la transmisión y el control de las Carreras Rituales. Los grupos de residencia

4 Otros cantos danzados con características similares son los buñua, pero estos no se interpretan durante los bailes de Yuakt. 
son determinados por estas líneas de transmisión de la Palabra5 ${ }^{5}$ La exogamia obliga a que los miembros de un grupo busquen sus esposas fuera del grupo de consanguíneos, lo que no siempre resulta fácil dado que la estructura del sistema de parentesco hace que se produzca una cantidad importante de parientes, limitando las nuevas alianzas en los grupos locales. J. Gasché (1976) ha demostrado que este fenómeno ha generado estrategias de disimulación de antiguos vínculos de parentesco con el objeto de producir aliados potenciales en el interior del grupo. Es suficiente esperar algunas generaciones para que dos grupos emparentados diluyan tal vínculo y puedan verse nuevamente como «afines potenciales», lo que lo lleva a calificar tal fenómeno como una suerte de illusion exogamique, es decir una suerte de endogamia disimulada. Ahora bien, según E. Viveiros de Castro,

à medida que passamos da area proximal às regiões distais do campo recional, a afinidade vai progressivamente prevalecendo sobre a consangüinidade, acabando por se tornar o modo genérico da relação social (Viveiros de Castro, 2002).

El parentesco se vuelve entonces menos eficaz a la hora de explicar las relaciones que superan lo local. El foco de análisis se desplaza así hacia las relaciones de afinidad pero no solamente aquellas ya confirmadas (afinidad real) sino que va más allá para dar cuenta de los grupos (humanos y no humanos) con los cuales es posible entablar tales relaciones, es decir el foco de análisis migra hacia la «afinidad potencial». El estudio de la afinidad potencial exige un tipo de herramientas distintas a las del parentesco, de manera que sea posible dar cuenta de vínculos en escenarios supralocales. Por tal razón nuestro enfoque le da un lugar privilegiado al análisis de las relaciones ceremoniales.

Un requisito fundamental para la celebración de un baile es la maloca, es decir que antes de convertirse en celebrante, un iniciado debe conocer los repertorios ceremoniales ligados a la construcción de este tipo de vivienda así como los aspectos que implican la vida en sociedad en este modelo de organización 6 . Hasta hace algunas décadas las malocas constituían la principal unidad de residencia; no obstante hoy su uso se reserva a las reuniones y fiestas colectivas. Algunos autores usan el término «grupo de residencia» para describir los integrantes de una maloca: la unidad compuesta por los miembros de las familias nucleares de un jefe (iyaima), las de sus hermanos (células), además de las hermanas solteras, los «huérfanos» (jaieniki) ${ }^{7}$ y antiguamente también los prisioneros ${ }^{8}$. En la percepción

5 El término «Palabra» es la traducción del término uitoto Uai que en ocasiones es traducido como «VOZ». Sin entrar en su complejidad, en este documento lo utilizaremos (con mayúscula inicial) para referirnos al conjunto de discursos y tradiciones que conforman el pensamiento uitoto.

6 Para un análisis sobre la maloca uitoto, cf. Corredor (1986).

7 Los "huérfanos" son individuos de otros linajes que sin tener una relación de alianza matrimonial con el grupo local ni un vínculo de consanguinidad se instalan en la zona de influencia de una maloca bajo la protección de un jefe.

8 En otro tiempo, en las guerras con los grupos antagónicos era habitual la toma de prisioneros quienes permanecían en la maloca hasta el momento en que eran sacrificados como parte de un rito antropofágico (Pineda Camacho, 2005). 
local un «grupo de residencia» es identificado con una maloca (salvo en los casos de escisiones de la maloca originaria donde un mismo «grupo de residencia» se distribuye en varias malocas9), y el conjunto de personas que conforman la maloca son aquellos llamados a integrar el grupo de anfitriones o nacoył (trabajadores).

En la lengua uitoto el término nacoył designa al conjunto de consocios que participan activamente en las labores de preparación de este tipo de ritos. Una característica determinante de este grupo es el hecho de que con ninguno de ellos pueden evolucionar alianzas matrimoniales: con los consanguíneos por el tabú del incesto y con los afines porque ya existe una alianza real con ellos. Las alianzas con los huérfanos (jaieniki) son desestimadas por ser vistos como de menor rango, pero son siempre posibles. Estas alianzas resultan notablemente particulares pues los huérfanos se hallan en una maloca ajena justamente porque su grupo de origen o ha desaparecido o se halla fuertemente distanciado. Tales circunstancias hacen que la alianza con un huérfano no represente un intercambio real con su linaje. Al contrario, actúan como miembros permanentes de sus malocas aliadas, convirtiéndose en una suerte de cognado, lo que explica por qué los matrimonios de huérfanos tienden a la uxorilocalidad (Gasché, 1976). Poco se conoce acerca de las relaciones con los prisioneros que integraban las antiguas malocas. De ellos se dice que, con cierta frecuencia, se les brindaba una esposa antes de ser sacrificados (Pineda Camacho, 2005). Sin embargo, más que una alianza (intercambio) parecía ser una forma de integración al grupo, de modo que el ritual antropofágico adquiría una connotación sacrificial (Leach et al., 1980). De cualquier manera, los lazos o las relaciones con los linajes de estos prisioneros no se materializaban de manera alguna.

Los nacoył son los encargados de producir los alimentos destinados a la fiesta. Para tal fin consagran una gran chagra (jakafał) al cultivo de yuca brava (Manihot esculenta). Los hombres se encargan de la derriba y limpieza del campo de cultivo mientras las mujeres asumen la responsabilidad del cultivo y cuidado. En una chagra unifamiliar intervienen solo los miembros del grupo doméstico, salvo si se trata de trabajos de gran dimensión como la chagra de baile donde participan los miembros de una maloca («grupo de residencia»). La recolección de la yuca destinada al baile se hace en una sola jornada por lo que también participa el conjunto de los miembros de la maloca celebrante; se trata de un trabajo de gran exigencia pues en un baile participan varios cientos de personas. Luego las mujeres de la maloca se ocupan de la producción de los alimentos, tamales de yuca así como de la cahuana (jargabł̇)10. A diferencia de la mayoría de las celebraciones de la Amazonía central y oriental, en estas fiestas colectivas no se producen bebidas fermentadas de ningún tipo. Los hombres de la maloca participan eventualmente de la producción de alimentos; no obstante su actividad

9 Salvo en los casos en que se especifique, en adelante utilizaremos el término «maloca» para referirnos al «grupo de residencia».

10 La cahuana (jałgabí) es una bebida preparada con almidón de yuca usualmente endulzada con jugo de frutas. 
fundamental es la producción de mambe11 y ambíl12 así como los trabajos de reparación de la maloca.

\section{2. Los visitantes}

El segundo tipo de participantes son los visitantes quienes se ocupan de abastecer la celebración con frutos y carne de animales salvajes así como de preparar y ejecutar los cantos-danzados (zaite) que constituyen el eje de las actividades de este tipo de celebración. Cuatro son los roles posibles de los grupos invitados: muruikit, muinakí, jayoki y jimukí. Cada rol está determinado por la situación de la maloca: así un bailarín deberá asumir el rol muruikí cuando integra los bailes situados al este de su residencia, muinaki cuando la maloca celebrante está al oeste, jimukí cuando está relativamente al sur y jayokí cuando la maloca celebrante se sitúa relativamente al norte. En cada caso los grupos de baile están liderados por el grupo de baile de la maloca aliada. Un celebrante deberá contar con cuatro malocas aliadas si desea celebrar un baile con la intervención de grupos de todos los roles. Sin embargo hoy estos bailes son más escasos contentándose con bailes donde participan dos o tres de los grupos ya citados.

Cada maloca aliada lidera los grupos de baile que asumen un mismo rol; el jefe de esta maloca deviene fuerama o «contendor» de la maloca celebrante. Los contendores aseguran los cantos más importantes de su grupo y se ocupan de coordinar las faenas de caza destinadas a garantizar el intercambio ceremonial de alimentos durante la fiesta. El rol de fuerama se prolonga hasta el fin de la carrera ritual del celebrante, es decir que estos no cambian durante toda la sucesión de bailes que implican estas carreras. Los contendores son seleccionados entre los jefes de las malocas con quienes no existe ni alianzas matrimoniales ni lazos de consanguinidad. Estos pueden pertenecer tanto a los clanes13 uitotos como a cualquiera de los grupos de la Gente de Centro; es decir que los contendores (y en general los visitantes) se derivarían de los grupos que los miraña califican como la «Gente de Dios».

11 El mambe (jíibie) es el polvo obtenido después de tostar, pilar y cerner las hojas de coca con adición de ceniza de hojas de yarumo (Cecropia sciadophylla).

12 El ambíl (yera) es el extracto espeso de tabaco obtenido de la cocción del zumo de hojas de esta planta. Este es consumido luego de mezclarlo con sal vegetal.

13 Llamaremos clan al conjunto de familias asociadas por un vínculo de consanguinidad determinado por la línea paterna. Los clanes son asignados de manera indiferente a los hijos de ambos sexos. Estos grupos son fundamentalmente exogamos y guardan un vínculo con ciertas especies animales y vegetales. No obstante los uitotos no le atribuyen a estos seres el caracter de ancestro: los uitotos afirman que los clanes derivan de la anaconda originaria, la cual fue segmentada y comida por cada grupo. La parte de la anaconda que le correspondió a cada uno de los jefes que participó de esta vianda, o la manera como estos recibieron su parte de la anaconda definieron el nombre de cada clan. El número exacto de clanes uitotos es difícil de determinar sobre todo porque su modo de parentesco presenta ciertas particularidades que les permite generar nuevas líneas de descendencia (cf. Gasché, 1976) 


\section{2. 1. Muruiki y Muinaki}

Los murui y los muinane14 son dos de los subgrupos uitotos más relevantes. Los primeros están situados fundamentalmente en la vertiente del río Caraparaná mientras que el segundo habita principalmente sobre el río Igaraparaná15. Los grupos murui y muinane se diferencian el uno del otro por su dialecto, pero también por la forma de sus malocas: las malocas muinane, definidas como «hembras», son soportadas sobre cuatro estantillos en una configuración rectangular; las murui, malocas «machos», comportan un estantillo adicional ubicado en la parte central. Esta característica masculina (fuerte) y femenina (dócil) define también el carácter y el comportamiento de estos grupos.

Los bailarines muruikt, provenientes del oeste, danzan siempre portando un bastón (toiraí) como elemento distintivo de su atuendo. Los cantos de los grupos muruikt con mucha frecuencia toman la forma de adivinanzas (eeiki bitaja): utilizando metáforas, onomatopeyas y pasajes de ciertos mitos los invitados componen acertijos con los que indagan por los animales o por las frutas traídas por ellos mismos. Estos acertijos buscan confirmar los conocimientos del celebrante sobre aspectos ceremoniales, sobre la historia natural o sobre la mitología de su pueblo. La enunciación de este tipo de adivinanzas, más que un juego, es un reto donde se pone a prueba al celebrante quien, con cada respuesta, se «defiende» y legitima (Gasché, 2007). Hasta hace algunos años, dicen los uitotos, un jefe incapaz de responder a estas adivinanzas corría el riesgo de perecer antes de terminar el baile, lo que muestra el carácter antagónico de este y de los demás grupos de baile.

En lugar de bastones los bailarines muinaki, provenientes del este, portan helechos que dentro de las actividades chamánicas uitotos son usados como medio de purificación. Los muina, dice Mogoratoł́, un anciano uitoto:

son grandes curanderos, no buscan ni siguen los problemas que causan daño a la población, no guardan rencor ni soberbia, su corazón es tolerante y lleno de bondad, porque son médicos tradicionales (Candre, 2011).

Al contrario de los muruiki, los cantores muinaki entonan «cantos sencillos, alegres y emocionantes». En estos cantos las adivinanzas son más escasas y tienden a ser menos complejas, lo que no impide, sin embargo, que actúen siempre como antagonistas de la maloca celebrante.

\section{2. 2. Jimuki y Jayoki}

Los jimuki son el tercer de los cuatro roles que pueden asumir los invitados de un baile de Yuaki. Contrario a los muinaki y muruiki estos no evocan ninguno de los subgrupos uitotos sino a un grupo externo. Con el término jimoki o jimuki los uitotos designan, en primer lugar, a una especie de primate de la familia Aotidae

14 El término Muinane se usa también para referirse a otro de los pueblos de la región que habla una lengua más próxima a la lengua Bora que a la lengua Uitoto (Londoño Sulkin, 2004).

15 Además existen otros subgrupos dialectales entre los uitotos tales como los nipode, pero actualmente se hallan fuertemente disminuidos (Griffiths, 1998). 
y del género Aotus, pero también a los indígenas yaguas (Burtch, 2008a). Este grupo del noroeste amazónico cuya lengua pertenece a la familia Peba-Yagua habría poblado el actual territorio de los uitotos manteniendo guerras implacables con ellos. Las versiones actuales coinciden en afirmar que los uitotos habrían enfrentado a este pueblo expulsándolo del Igaraparaná luego de una feroz guerra (Teteye \& Teteye, 2005). Esto parece haber sido registrado por los relatos donde se les atribuye un trágico final a causa de su mal comportamiento: los yaguas habrían sido sumergidos en el fondo del río Igaraparaná. A través de su comportamiento, sus cantos y sus trajes, los jimuki, evocan la presencia de los antiguos yaguas16.

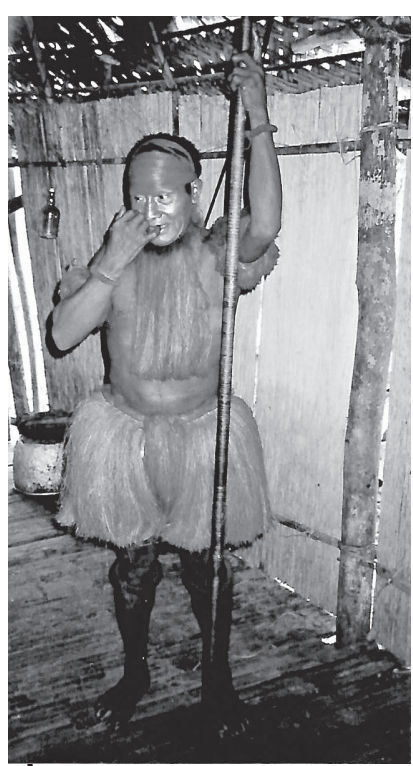

Figura 2 - Hombre yagua (Alto Atacuari) (1994)

(C) Bonnie Chaumeil

Los atuendos de este tipo de bailarines, notablemente similares a los de los ritos yaguas (Chaumeil, 2000), comportan faldas hechas de fibras de palma de especies como la Palma Real (Attalea butyracea) o la Palma de Cumare (Astrocaryum chambira). También usan tocados en la cabeza y un bastón de baile que lleva atado en la punta un manojo de fibras de la misma planta. En todos los casos los bailarines pueden maquillarse el cuerpo o adornarlo con plumas, pero estos no son rasgos característicos de un grupo concreto sino de los participantes en general (incluso los anfitriones).

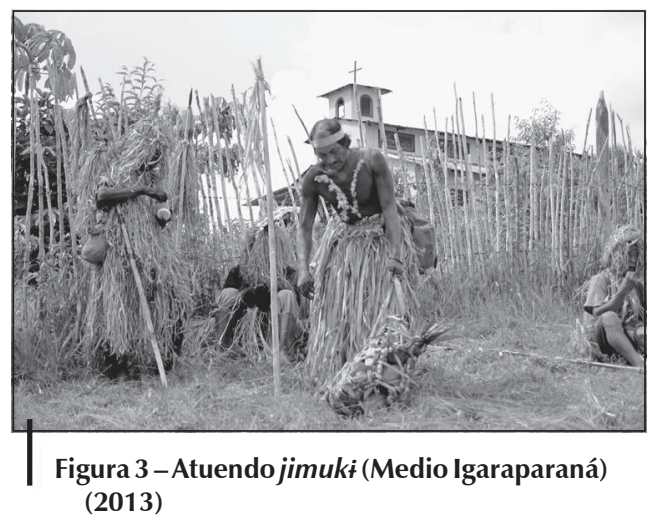

(C) O. I. García

Por otra parte tenemos a los bailarines jayoki, el cuarto y último de los roles posibles que pueden asumir los invitados al baile de Yuakt. El término jayoki está ligado a la palabra jaio (serpiente), pero también es el término con el que se refieren a uno de los pueblos de la región del río Caquetá. Los relatos locales cuentan que hasta

16 Algunos indicios apuntan a que el complejo de grupos al que hoy llamamos yaguas no sería el mismo que integraba esta nación hace algunos siglos (cf. Goulard, 2011; Chaumeil, 2000) lo que podría implicar que los relatos uitotos se referirían a un grupo diferente al que hoy conocemos con el mismo nombre. 
finales del siglo XIX existió un grupo que habitaba en las inmediaciones del Medio Caquetá: los Nayent (Gente de río) ${ }^{17}$. Sobre este grupo son pocos los datos que se conocen. Dos son los medios que mejor describen este pueblo: en primer lugar la tradición oral de los pueblos de la Gente de Centro (Kuyoteka \& Restrepo, 1997); en segundo lugar las prácticas ceremoniales de estos pueblos. Los relatos uitotos hablan de dos héroes míticos, los hermanos Jaidotroki y Nayenureye, quienes eran dos jefes de los grupos de la región. Del primero descendería la gente Jaijoni (Gente serpiente) y del segundo la gente Nayent (Gente de río). Los Nayeni habrían habitado en la rivera izquierda del río Caquetá cerca de uno de los raudales próximos a la comunidad de Araracuara; allí también habrían desaparecido por completo luego de un aparente desastre. Dicen los lugareños:

Los Jaijoni "(...) se organizaron para hacer baile de palma jaijoma. La historia original de esa tribu jaijoní, [también llamada] 'gente burletera' (sic), que en otra forma se les dice 'jäjoma' que es 'tribu de culebra'. Ese era el nombre más usual para nominar a esta tribu. [Se les llamaba] la legión náyeni, pero el nombre legal [es decir el nombre verdadero] es jaijoni. [Las personas de ese grupo] no hablan bien... en forma desarrollista [es decir conforme a la Palabra ritual], [las personas de este grupo] profesan [todo lo] contrario a lo legal [a lo que instaura la Palabra ritual]. Por eso se les dice jayoma: [se les llama] raro, [en nuestros términos se les dice] eire úrite (Kuyoteka \& Restrepo, 1997: 409 § 24).

Los trajes de este grupo se caracterizan por el uso de faldas de chambira (Astrocaryum chambira) que van un poco más abajo de la rodilla. No obstante actualmente el rasgo más característico es el uso de ramas de palmas de entre dos y tres metros de largo de la misma especie con los cuales danzan durante toda la ceremonia. En algunos casos usan bandas atadas a los brazos o a la cabeza pero como en el caso del maquillaje no son rasgos exclusivos de estos invitados. Por otra parte sus cantos son también caracterizados por la importante presencia de adivinanzas.

\section{3. Un sistema biaxial}

El baile pone en evidencia la expansión progresiva de las relaciones sociales partiendo de la maloca (comunidad celebrante) y extendiéndose por un eje fluvial (este-oeste) a los subgrupos murui y muinane. Un segundo eje relativamente perpendicular completa este modelo vinculando el sector norte y sur del territorio. En términos espaciales, al norte del territorio uitoto se sitúan los grupos Nayenł (y carijonas18) y al sur los yaguas, razón por la cual este eje sirve de identificación a los invitados jayoki y jimuki respectivamente.

17 Los muinanes los Ilaman Nifaimina (Gente de Agua) describiéndolos también como grupos enemigos y atribuyéndoles un fin similar (Consejo de Ancianos de la etnia muinane, 1996).

18 Los carijonas hablan una lengua caribe. Estos eran célebres por el comercio de esclavos y por su carácter guerrero y habrían sido el principal impedimento de la expansión de los pueblos de la Gente de Centro hacia el norte del río Caquetá. 
Estos dos ejes vinculan entonces grupos de características diferentes. El eje horizontal está dedicado a los subgrupos uitotos que, en tanto afines, son vistos como una suerte de enemigos próximos. El segundo eje está dedicado a las relaciones con los grupos decididamente antagónicos con los que los intercambios son notablemente limitados (enemigos lejanos); estos definen un grupo de alteridad que si bien en las danzas es solamente evocado, como veremos, no lo hace sino a través de un dispositivo de representación. En el contexto del baile, esta correspondencia espacial definida por los ejes es siempre relativa al centro del sistema (la maloca celebrante). Solo hasta cuando se identifica la maloca anfitriona es posible identificar quienes asumirán los roles muruiki, muinakit, jimukí o jayoki.

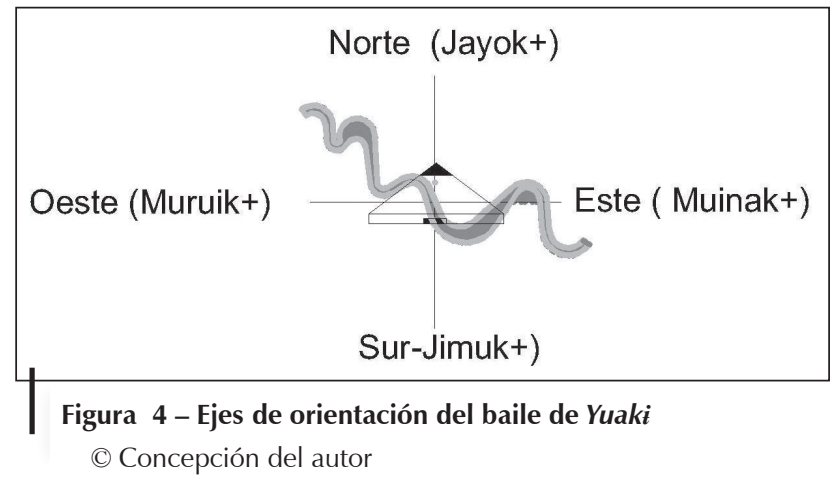

Una de las partes más importantes del Baile de Yuaki es la ceremonia de entrega de cacería donde tienen lugar un conjunto de danzas a través de las cuales se confirman estos dos ejes. Durante esta parte de la ceremonia los bailarines muinaki ingresan a la maloca con pasos relativamente cortos alternando desplazamientos hacia adelante y hacia atrás con pivotes frecuentes. Normalmente el pie derecho avanza y retrocede con pasos cortos acentuando un poco más el movimiento que el pie izquierdo. Este último avanza siempre con pasos cortos. En los dos casos la planta del pie hace contacto total con el suelo. El tronco permanece ligeramente inclinado (a veces redondeado y a veces ligeramente contraído) así como las rodillas ligeramente flexionadas. Los bailarines portan ramas de helechos en una o en las dos manos, los cuales son agitados hacia el exterior en un movimiento que coincide con el del pie derecho. Los brazos se mantienen paralelos salvo por las variaciones que implican los desplazamientos laterales. Por su parte, los bailarines muruiki se valen de pasos cortos con ligeros desplazamientos laterales. El ritmo de su intervención cambia pues en lugar de la cadencia del movimiento de los helechos estos acentúan los pasos con los golpes del bastón de ritmo contra el suelo. En este caso el brazo que porta el bastón se mantiene ligeramente flexionado describiendo a la vez un movimiento progresivo que hace que cada golpe impacte un lugar diferente como un arco perpendicular a la línea de desplazamiento (una suerte de abanico). Como en el caso anterior el tronco y la cabeza intervienen escasamente. En ocasiones los bailarines dan pasos hacia atrás pero en general el movimiento es hacia adelante. 
La danza de los bailarines jimuki y jayoki muestra también coincidencias coreográficas entre ellas. Los bailarines jimukise desplazan de manera relativamente lenta privilegiando el nivel bajo. Con frecuencia intercalan su desplazamiento con breves paradas en posición arrodillada o en cuclillas. Estos prefieren avanzar a través de movimientos laterales pivoteando siempre con su bastón de ritmo. En este tipo de danza los cambios de frente son mucho más frecuentes que en los dos casos precedentes. Estos desplazamientos laterales son intercalados con eventuales, pero escasos, desplazamientos frontales y con repentinos saltos apoyados en el bastón. Aun si los brazos permanecen cerca del eje del cuerpo se trata de un desplazamiento mucho más abierto gracias a la configuración que generan los desplazamientos laterales, los pivotes y los saltos de bastón. Finalmente tenemos las danzas jayoki que, como en el caso jimukí, utilizan pasos normales o largos. En general los pies y los brazos permanecen alejados del eje corporal lo que le da un carácter relativamente abierto. Como en el caso anterior la velocidad de desplazamiento es relativamente lenta privilegiando los movimientos hacia los costados. Junto con esto este tipo de danza opta por los desplazamientos a un nivel relativamente bajo. Contrario a las coreografías muinaki y muruiki estas dos últimas generan movimientos de brazos simultáneos dirigidos por los artefactos de baile, lo que es menos frecuente en los casos anteriores aun si la danza se sirve del bastón de ritmo. A través del movimiento de las palmeras los bailarines jayoki hacen una suerte de barrido en abanico rozando la hojas de la rama contra el piso. Este hecho, dicen los uitotos, figura el desplazamiento de una serpiente, lo que coincide también con la danza jimuki a la que los lugareños atribuyen figuraciones ligadas a los monos que representan tales como los saltos.

La dirección y la forma de los movimientos en los cuatro grupos. Pese a ello, es posible ver que en los casos muruiki y muinaki existe una tendencia hacia los pasos cortos (o muy cortos) a velocidades relativamente mayores que las de los otros dos grupos. Los gestos muruiki y muinaki cambian de nivel con poca frecuencia manteniéndose en el plano medio o bajo de manera permanente hasta el fin del performance. Al contrario las danzas jayoki o jimuki presentan cambios de nivel pasando del nivel medio al nivel bajo con relativa facilidad. En cuanto a la extensión de los movimientos es posible ver que los dos primeros grupos privilegian los movimientos cerrados, manteniendo los brazos y piernas muy cerca del eje del cuerpo a diferencia de los otros dos grupos que privilegian los movimientos abiertos con una mayor separación de brazos y piernas respecto al eje del cuerpo. Por otro lado los desplazamientos de los grupos muruiki y muinakt tienen una tendencia frontal mientras que los de los jayoki y jimuki privilegian el avance hacia los costados generando grandes diagonales que les permiten intervenir mayormente en la escena de baile.

Las danzas confirman la proximidad entre los grupos muruiki y muinaki de un lado, y entre los grupos jayoki y jimuki del otro. Tal proximidad Ilama aún más la atención sobre los dos ejes (perpendiculares) que estos grupos conforman. Además algunas interpretaciones sobre las malocas de la Gente de Centro —en tanto construcción — dan cuenta de la existencia de un eje que une las entradas 
anterior y posterior, evocando un río imaginario que lo atraviesa de un lado al otro dividiéndolo en dos sectores (Karadimas, 2005). La tendencia a los movimientos frontales de los bailarines muruiki y muinaki coincidiría con este eje fluvial imaginario mientras que los desplazamientos abiertos y hacia los costados de los grupos jayoki y jimuki lo harían con un eje perpendicular a este mismo río. Este sería un nuevo argumento a favor de un modelo relacional biaxial que reuniría tanto elementos de carácter espacial como de orden social.

Hasta aquí hemos visto cómo la maloca se amalgama bajo un principio de identidad cognaticia (real o simbólica) que define un sistema que comporta una geometría biaxial a partir de la cual se describen relaciones de alteridad. En estas descripciones hemos introducido rangos de mayor o menor proximidad entre los grupos. No obstante para comprender tales distancias es preciso analizar uno de los aspectos cruciales de este tipo de economía simbólica de la alteridad: la circulación. Por tal razón es necesario concentrarse en los elementos simbólicos que son intercambiados durante el baile.

\section{LA CIRCULACIÓN EN EL SISTEMA}

Partiendo del análisis del baile como dispositivo terapéutico es posible mostrar cómo tienen lugar los intercambios entre humanos y no humanos. Estos intercambios son ante todo simbólicos y se rigen por una lógica de depredación. Los mismos resultan de un impacto tal que logran determinar la distancia entre los grupos sociales (humanos y no humanos) que conforman el sistema de organización social uitoto.

\section{1. El baile como terapia 19}

El modelo nosológico uitoto reconoce buena parte de las «enfermedades exógenas» tales como las enfermedades infecciosas, parasitarias, tóxicas, traumáticas, e

19 Los uitotos describen ciertas patologías como de carácter colectivo. Algunas son de carácter psíquico y otras son desordenes morales; a todas se les atribuye la capacidad de desplazarse como un objeto material que es enviado por los animales y entidades espirituales antagonistas. Las acciones desarrolladas durante el baile se orientan al tratamiento de la colectividad (la maloca) como un macrocuerpo, de modo que las acciones de «curación» incluyen tanto prevenciones ligadas a la gnoseología como a los consejos que buscan resolver desordenes morales o sociales. Incluyen también acciones que buscan neutralizar las fuentes y agentes que envían estas enfermedades. En este artículo hablamos de «terapia» y no de «curación» para referirnos al acto orientado al restablecimiento de la salud del macrocuerpo que es la maloca y su zona de influencia (los uitotos aseguran que los efectos terapéuticos del baile tienen un límite fijo y visible: el lugar hasta donde llega el tabaco de invitación). El término «curación» parece menos incluyente dado que la presencia de estos desórdenes no implica la presencia de la enfermedad en el cuerpo del paciente (individuo), sino que se trata de un entidad con presencia latente que afecta potencialmente al colectivo. El término «terapia» reconoce también la enfermedad como entidad independiente y no solo como la reacción fisiológica de un paciente. La concepción de la enfermedad entre los uitotos, pone en evidencia la amplitud de las actividades de los especialistas rituales (chamanes) y lo que para ellos 
incluso alérgicas. A esta categoría los uitotos adicionan sin embargo, otro tipo de desórdenes tales como la rabia, la envidia, el aburrimiento, los celos y la locura. Estas patologías adicionales son atribuidas a agentes externos como los espíritus de los animales, las plantas u otras entidades espirituales20. Los uitotos afirman por ejemplo que la «rabia» puede haber sido causada por los espíritus de las hormigas quienes vengarían la pérdida de sus «malocas» en los habituales incendios destinados a la agricultura indígena. Los «celos», ciertos tipos de «pereza» así como algunos desórdenes sexuales son atribuidos a una entidad espiritual Ilamada jabo rł́ngo (falsa mujer); la «locura» (sic) es atribuida al murciélago. La fuerte capacidad de propagación que se le atribuye a enfermedades enviadas por animales y plantas justifica los procedimientos o terapias colectivas. Solo la eliminación de la fuente o el redireccionamiento de la circulación de estas acciones patógenas evitará la reincidencia o la propagación de las enfermedades. Los uitotos declaran que el objetivo mayor de los bailes de Yuaki, es la búsqueda de la fuente de los «aires» de enfermedad. A través del uso de discursos performativos, durante estos bailes, los «aires» de las plantas silvestres son resituados al interior de la selva (lejos de la comunidad) y los animales agresores son transformados en mujeres. Luego estos animales son llevados a la maloca celebrante donde son intercambiados por productos derivados de la yuca (cocidos). Dado que las mujeres uitotos se identifican con la yuca y que los animales traidos han sido previamente convertidos en mujeres, la cacería de estos bailes puede interpretarse como un intercambio de mujeres. Un intercambio mediado por la guerra como el que tiene lugar entre los grupos yaguas (cf. Chaumeil, 1985). Al final, los chamanes, se apoderan de las armas de estos animales (colmillos, garras, plumas, etc.), mientras que las personas de la maloca se quedan con la carne de estos agresores que eran fuente de enfermedad. Las ceremonias de entrega de la caza que veremos a continuación ilustran el momento del intercambio antedicho.

\section{1. 1. Rito de entrega de caza}

Como parte de los preparativos del baile, el celebrante hace la ceremonia de invitación, en la cual a través de un discurso performativo, transforma las actividades de caza en intercambio de mujeres. Aquí cuatro actores resultan fundamentales: el jefe anfitrión (rafue naama), el jefe contendor (fuerama), el emisario (yoraima) y el cazador (raoraima). El primero (rafue naama) mediante un discurso ritual consagra el tabaco de la invitación donde transforma los animales

es la acción de «curar». Como vemos, para los uitotos, las actividades que buscan el mantenimiento adecuado del estado de salud de las personas implican un conjunto de prácticas que van más allá de la prevención-saneamiento-recuperación. En este sentido, la gestión de las relaciones entre humanos y no humanos desborda el sentido del término «curar». Agradezco a S. Hugh-Jones por las críticas relacionadas con este y otros aspectos tratados en este texto.

20 Otras enfermedades atribuidas a los animales derivan de su consumo: algunas resultan del exceso en la ingestión de ciertas especies, otras obedecen a tabúes ligados a la edad o al patrilineaje: cada grupo patrilineal es asociado a una especie animal o vegetal, lo cual puede generar un veto al consumo de tales especies relacionadas. 
agresores en mujeres. El segundo (yoratma) transporta este tabaco a casa del contendor (fuerama); este lo redistribuye entre los cazadores (raoraima) de su maloca quienes a su vez lo llevan ante las entidades espirituales de los animales (dueños) para intercambiarlo por cacería (mujeres transformadas). Teniendo en cuenta que entre los uitotos los animales son vistos como «gente», este tipo de actividad cinegética es replanteada: la depredación, en tanto caza, se convierte en guerra dado que la presa se convierte en enemigo. En este contexto el estatuto ontológico del depredador y la presa es equivalente: el animal deja de ser una presa para transformarse en enemigo.

El ambíl es consagrado a través del canto o la oración del tabaco (zomarafue21) el cual le transfiere un conjunto de capacidades performativas, entre otras la de cazar. Por tal razón a esta actividad se le llama la cacería del tabaco. Kinerał, un anciano uitoto, explicaba así el funcionamiento de este tipo de cacería:

la cacería de tabaco es cacería de todo animal y es del trabajo de chagra. Cuando se hace el tabaco [el ambíl] y se nombra el animal, ese cae ahí, ese es cacería de tabaco. Cuando uno quiere hacer cacería mezcla ambíl y nombra y ahí cae (Candre-Kinerai \& Echeverrí, 1993).

En efecto, la muerte de los animales no es atribuida a los cazadores del fuerama sino a la acción del zomarafue con el que se ha consagrado el tabaco del baile. Los miraña coinciden con esta creencia. Ellos aseguran que son las historias (la Palabra) las que tienen el potencial de actuar; la preparación no es sino el contenedor de este potencial (Karadimas, 2004).

Luego de que el fuerama recibe el tabaco enviado por el celebrante, este es distribuido a los cazadores de su maloca quienes parten en busca de los animales sentenciados a muerte por el rafue naama. Al término de estas faenas de caza, estos se presentan en la maloca vestidos con sus trajes característicos para hacer entregar de los animales abatidos. Esta entrega se hace a través de cantos llamados uuriya rua (cantos de llegar alegremente) o fakariya rua (cantos de llegar para probar). La primera acepción evoca el júbilo que representan los bailes y la segunda el hecho que los cantores llegan a «probar» (facade) los alimentos que se preparan para la fiesta, particularmente las bebidas a base de yuca.

Cada cazador llega a la maloca cantando y danzando desde el exterior. Cuando ingresan los conayi (generalmente hombres) reciben la cacería traída; luego una o dos mujeres anfitrionas se aproximan a cada bailarín con calabazas Ilenas de jugo de manicuera (yuca dulce)22. Allí se genera una tensión entre los bailarines

21 Este término deriva del verbo zomade (cantar en solitario para enseñar a los otros) y rafue que en este contexto de refiere a la Palabra del Baile. Se trata de una larga recitación basada en paralelismos y en la que la condensación ritual tiene lugar en el momento mismo de la enunciación. El análisis de este aspecto desborda el objetivo de este artículo pero para ver un análisis de un ritual con acciones rituales similares puede consultarse, Severi $(1993 ; 2002 ; 2007)$.

22 La manicuera (Manihot esculenta) es una variedad de yuca particularmente dulce consumida como bebida. Entre los uitotos la manicuera o yuca dulce se halla vinculada frecuentemente a las representaciones femeninas. 
y las mujeres; las primeras intentan frenar su arribo al mambeadero mientras los cazadores hacen lo posible para evitarlas sin dejar de cantar y bailar. Esta acción continúa sin interrupción hasta que ellas logran hacerlos beber el jugo de manicuera. Se trata de un acto de purificación atribuido a la yuca dulce a la cual se le presta la capacidad de restituir el estado de equilibrio provocado por ciertos desórdenes como la rabia, lo que en el contexto ceremonial es llamado «endulzar». Luego de beber el bailarín continúa cantando en dirección del anfitrión delante de quien canta y a quien le plantea el eventual acertijo que porta su canto. En presencia del celebrante la acción de purificación iniciada por las mujeres se completa cuando el espíritu del animal cazado por el tabaco entra definitivamente en un calabazo de tabaco líquido (yerako), consagrado por el celebrante para este fin desde el comienzo de los preparativos del baile. Así se cierra la trampa o dispositivo terapéutico que liga los animales con el baile. Luego el rafue naama descifra el acertijo traído por el bailarín y después este es retribuido con casabe, envueltos de yuca y cahuana (jugo de yuca amarga): así, los animales traidos (mujeres transformadas) son intercambiados por yuca, la cual, en el contexto uitoto, identifica a la mujer.

La creencia sobre la capacidad de curación del baile (eficacia) estaría ligada al vínculo existente entre animales y enfermedad: por esta razón cuantos más son los animales traídos a la maloca, más fuerte es la creencia sobre la capacidad del celebrante. De manera simultánea los cazadores ganan una cuota de prestigio al «traer» tal cacería, de modo que extremarán sus esfuerzos para lograr un buen resultado. Es un dispositivo notablemente eficaz que une las nociones de saludenfermedad con las de prestigio para asegurar el éxito de esta acción ritual. No obstante, si a los cazadores no se les puede atribuir el fruto de la cacería, ¿de dónde proviene su prestigio? Como lo hemos dicho, en el contexto de esta caza ceremonial los cazadores no actúan como depredadores sino como una suerte de presa o mejor como una trampa que atrae estos agentes patógenos expulsados por los animales. En efecto, cuando los cazadores «traen» los animales cazados por el tabaco no pueden evitar contaminarse con tales agentes. Esta sería la razón por la cual durante estos bailes los nayení y los yaguas son representados como jimuki y jayoki respectivamente: en tanto enemigos, los cazadores jimuki y jayokt estarían más propensos a atraer agentes patógenos como las rabias enviadas por los jaguares, para portarlas luego a la maloca.

\section{EL BAILE DE YUAKł O LA DEFINICIÓN DE UN MODELO CONTINUO DE RELACIONES}

El baile de Yuaki da cuenta no solo de un modelo de relaciones desde el punto de vista uitoto sino del resto de la Gente de Centro. En efecto, este tipo de rito existe en las tradiciones ceremoniales de todos los pueblos de la Gente de Centro: los boras lo llaman apújko, los okainas Odyááhto, los nonuyas Jojał, etc. Cada grupo cuenta con sus propias coreografías y cantos, lo que les permite generar sus propias celebraciones y participar en las de los grupos vecinos. La proximidad entre estos 
pueblos no impide, sin embargo, la existencia de tensiones entre ellos. Los boras y los uitotos, por ejemplo, comparten la región del Medio Caquetá desde hace mucho tiempo, aun si sus relaciones parecen haber estado caracterizada por la guerra (Guyot, 1972: 148; Teteye \& Teteye, 2005). A pesar de lo anterior, los boras y los uitotos conservan y promueven intercambios matrimoniales, alianzas ceremoniales (bailes) e intercambios comerciales frecuentes. Al contrario, los yaguas y los nayent (Gente de los animales) no han actuado jamás en calidad de invitados (a pesar de ser representados en las danzas jimuki y jayokit). Las relaciones con estos grupos han sido siempre fuertemente antagónicas. Los yaguas, en particular, situaban a los uitotos entre los grupos con mayor grado de alteridad (munuñu), es decir aquellos calificados como «no gente» y por tanto «enemigos lejanos» con quienes no mantenían intercambios. Al contrario con ellos practicaba un tipo de guerra implacable donde las víctimas se extendían inclusive a mujeres y niños (Chaumeil, 1985).

El modelo de organización social descrito por el baile de Yuaki permite entonces desarrollar el modelo miraña estudiado por Karadimas. En los términos de Leach et al. (1980) se diría que el baile da cuenta de grupos situados en universos «exteriores próximos» (muruiki y muinakit) pero también de grupos que se hallan en «zonas intermedias» (Gente de Centro) y que coinciden con los grupos que el sistema miraña califica como «Gente de Dios». Así mismo este tipo de ceremonias da cuenta de universos «exteriores lejanos» (carijona, yagua, nayent, etc.), los cuales son o totalmente desconocidos o notablemente imprevisibles. Estos últimos corresponden con lo que en el modelo miraña se ha llamado «Gente de los animales».

Por otro lado, este baile pone en evidencia el dinamismo de la escala derivada de la noción de persona y del sistema de organización social uitoto. En efecto se trata de un modelo que no es ni estático ni exclusivo de los grupos humanos. Entre los uitotos, como en muchos pueblos del noroeste amazónico la equivalencia ontológica entre humanos y seres vivientes depende de su definición de persona:

todas las creaturas vivientes tienen el estatus de persona, el cual comprende diferentes grados: nuestras gentes son más personas que los enemigos y los extranjeros. Así la mayor parte de los animales son menos personas que la mayor parte de humanos y que ciertos animales. Los grandes depredadores salvajes, los herbívoros y los animales domésticos lo son más que sus congéneres más pequeños o menos domesticados. La equivalencia ontológica entre humanos y animales implica que las relaciones entre diferentes suertes de seres humanos, así como entre estos y los animales, no son más que una cuestión de grado (Hugh-Jones, 1996, nuestra traducción).

Entre los uitotos, uno de los elementos que determina la noción de persona está ligado a la «Palabra ritual». La mitología uitoto muestra que los grupos que albergan la «Palabra de Vida», es decir la que está asociada al Padre Creador, serían vistos como persona (Preuss, 1994; Kuyoteka \& Restrepo, 1997; Roman et al., 2010). Al contrario, aquellos que la poseen o no, o que poseen una versión impura como la «Palabra de los animales» se alejarían de este status (Roman et al., 


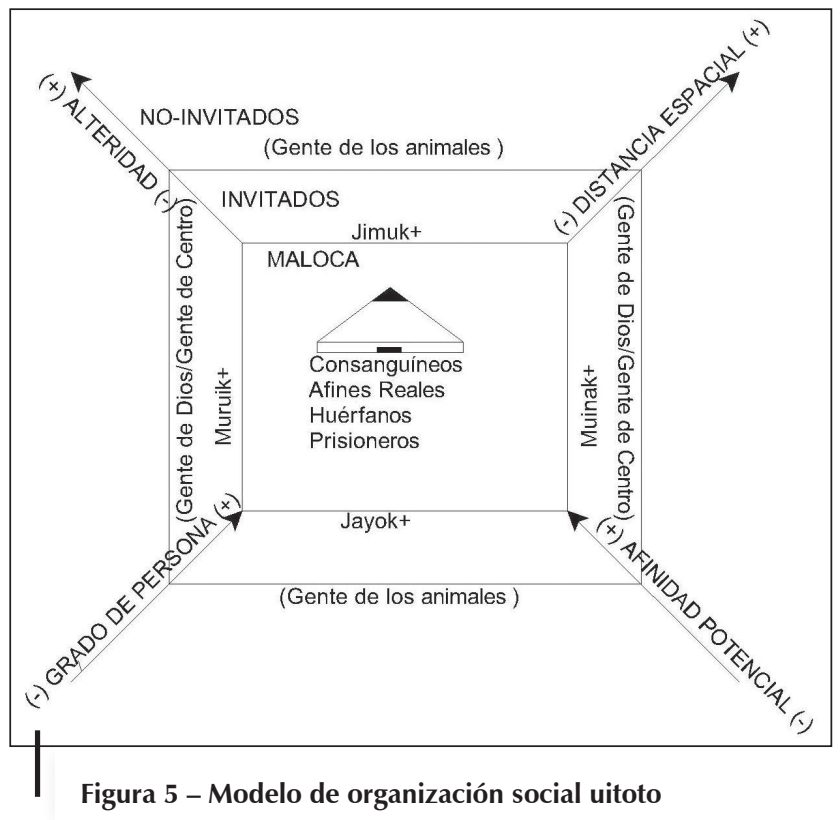

(C) Concepción del autor

2010). En los ritos de cacería que hemos discutido anteriormente el pago por los animales traídos es distinto si se trata de animales pequeños (roedores, peces o aves de corto vuelo) o si son grandes mamíferos y predadores solitarios como el jaguar o el águila. A los primeros se les atribuye un antagonismo derivado de la competencia que existe entre estos y los humanos por los productos de la chagra. En cambio, la enemistad con el segundo grupo deriva de su comportamiento antisocial y agresivo que evidencia el uso de una «Palabra impura» la cual los lleva incluso a depredar a sus congéneres (endocanibalismo). En esta escala los humanos no se sitúan en la posición que comporta el mayor «grado de persona», atribuido a ciertas entidades espirituales 23 , de la misma manera que al jaguar no se le atribuye la posición de menor «grado de persona» ostentada por otras entidades espirituales.

Los pueblos yukunas, makunas y tanimukas hacen parte de la Gente de los Animales, es decir de los grupos de «exteriores lejanos». Sin embargo, como lo muestra el análisis del baile de Yuaki, las relaciones de intercambios entre estos grupos y los uitotos los sitúan más cerca que los grupos yaguas y carijonas, pero mucho menos que cualquiera de los grupos de la Gente de Centro. En efecto, las relaciones con estos grupos no se definieron a través de guerras despiadadas como aquellas sostenidas con los grupos yaguas y carijonas donde a estos últimos

23 No obstante estas entidades son ambiguas porque en ocasiones sus actos los llevan de un extremo a otro pasando de manifestaciones de gran sapiencia a fuertes actos trasgresores. 
se les atribuía un estatus de persona próximo al de los grandes depredadores y cargado de un fuerte grado de alteridad 24 . Al contrario, estos grupos, poseedores de prácticas asociadas al uso de la Palabra ritual, tales como las danzas colectivas y el consumo de coca y tabaco, resultan menos distantes para los uitotos. Esto parece explicar la tendencia reciente a generar alianzas ceremoniales entre estos grupos y la Gente de Centro25. Estos contactos ceremoniales sugieren incluso un cambio de grado de estos grupos que parece llevarlos de los extremos de la escala de afinidad potencial al sector de la Gente de Centro, aumentando la posibilidad de pasar de afines potenciales a afines reales. El modelo yagua, dice Chaumeil, evidencia una aproximación equivalente entre los yaguas y los uitotos: desde la perspectiva de los primeros los uitotos habrían pasado de ser «enemigos lejanos» a «enemigos próximos» 26 . No obstante el modelo derivado del baile de Yuakt no refleja tal aproximación. Aun si yaguas y nayenis son vinculados con estas ceremonias, lo anterior no parece ser signo de un real intercambio. Al contrario se trataría más bien de una confirmación de la distancia de alteridad que los separa. Pese a ello, lo anterior no refuta la hipótesis de la aproximación entre estos dos grupos sociales pues los indicadores usados son ligeramente diferentes.

La sociedad no indígena (los blancos) también es vinculada a este modelo, incluso si su rol no siempre ha sido el mismo. Los blancos, así como los carijonas, eran llamados riá, término uitoto que deriva del verbo rite (comer carne). Esto a causa de las prácticas antropofágicas indiscriminadas atribuidas a los primeros y a la violencia brutal con la que se manifestaron los blancos durante los primeros contactos, en la época de la bonanza del caucho (1870-1932). Al final de este periodo histórico las relaciones con el blanco se transformaron, particularmente por la influencia de las misiones cristianas en la zona, que se ocuparon de promover la idea de que la mitología uitoto correspondía de manera «casiexacta» a la mitología cristiana. Justifica así el status de persona del blanco dada su capacidad de contener la Palabra. Esto parece haber influido en el aumento

${ }^{24}$ S. Hugh-Jones $(2011 ; 1996)$ afirma que los tucanos otorgaban un estatus similar a los carijonas, lo que podría apoyar la hipótesis de la mayor proximidad entre los pueblos de la región del Apaporis y los del Medio Caquetá.

25 Urbina Rangel (1997) ha reportado recientes alianzas ceremoniales entre los nonuyas y los yukunas. Estos últimos, en calidad de «huérfanos», se habrían iniciado en la vida ceremonial nonuya llegando incluso a celebrar algunos de sus bailes rituales. Un fenómeno similar parece tener lugar en las proximidades de la ciudad de Leticia (Colombia) donde los uitotos conviven con los yukunas, makunas y matapis desde hace algunas décadas y donde es relativamente habitual ver que los unos participan de los bailes de los otros

26 Por otro lado, en las ceremonias yaguas los uitotos no parecen haber sido vinculados de una manera equivalente con aquella utilizada por los uitotos para dar cuenta de sus relaciones con los yaguas. De hecho, afirma Chaumeil, hasta hace poco no se reportaban representaciones uitotos en el contexto de los rituales yaguas. Es verdad que en ciertas regiones habían sido reportados casos de matrimonios de hombres uitotos con mujeres yaguas. No obstante es difícil calificarlas como tendencias. Por otro lado, las condiciones del desplazamiento después del conflicto del caucho, así como opciones alternativas de alianza como la que se define con el término «huérfano» entre los uitotos parecen reorientar estas hipótesis. En efecto, se trata de una investigación sobre la que la etnología de la región ha avanzado poco pero que empieza a abrir nuevas perspectivas de análisis, tanto en los aspectos sociales como económicos y ceremoniales. 
de los matrimonios, notablemente de mujeres indígenas con hombres blancos. Así en pocas décadas el blanco pasaría de uno de los puntos de mayor distancia de alteridad al de la afinidad real. Este pasaría de ser una suerte de «jaguar» para integrar los grupos conaył (cognado) al término de un periodo relativamente breve. En cuanto a los carijona, las relaciones para con ellos han cambiado de manera substancial: en la actualidad su presencia en la zona de influencia uitoto es casi nula, pero estos parecen haber sido introducidos en la vida ceremonial a través de una estrategia similar a la de los nayeni. Hoy los uitotos celebran un baile llamado «baile de Carijona», lo que parece desplazarlos en la escala de alteridad, pero la poca atención prestada a este y los demás bailes rituales de la región impide aproximarse a una conclusión más definitiva27. En la actualidad, aun si los estudios sobre el chamanismo han ampliado el horizonte sobre este tema, las investigaciones consagradas al ritual, tanto como a las danzas del noroeste amazónico continúan siendo insuficientes. Hasta hoy, son raros los estudios sobre las danzas así como los análisis comparativos que dan cuenta del grado de proximidad de las prácticas de intercambio ritual de estos grupos, incluso si muchos de ellos comparten elementos rituales (máscaras, cantos y danzas rituales). Tal fenómeno pone en evidencia la urgencia de insistir y profundizar en los estudios sobre los bailes y danzas rituales de los pueblos de la región tanto como en sus implicaciones y dinámicas sociales.

\section{Referencias citadas}

BURTCH, S., 2008a - Diccionario Huitoto-Murui, vol. I, 262 pp; Lima: Instituto Lingüístico de Verano (Serie Lingüística Peruana, 20).

BURTCH, S., 2008b - Diccionario Huitoto-Murui, vol. II, 166 pp; Lima: Instituto Lingüístico de Verano, (Serie Lingüística Peruana, 20).

CANDRE, A., 2011 - Historia de mi padre Mogoroti 'Guacamayo azul': palabras del ritual de las frutas que llega a nosotros como comida en abundancia, de parte de la etnia ocaina. Mundo amazónico, 2: 307-327.

CANDRE-KINERAI, H. \& ECHEVERRÍ, J. A., 1993 - Tabaco frío, coca dulce, 286 pp.; Bogotá: Colcultura.

CHAUMEIL, J.-P., 1985 - Échange d'énergie : guerre, identité et reproduction sociale chez les Yagua de l'Amazonie péruvienne. Journal de la Société des Américanistes, 71: 143-157.

CHAUMEIL, J.-P., 1994 - Los yagua. In: Guía etnográfica de la Alta Amazonía (F. Santos Granero \& F. Barclay, eds.): 181-308; Lima: Instituto Francés de Estudios Andinos, Flacso sede Ecuador.

CHAUMEIL, J.-P., 2000 - Voir, savoir, pouvoir : le chamanisme chez les Yagua de l'Amazonie péruvienne, 349 pp.; Genève: Georg. Éditeur.

27 Una primera aproximación al baile de los carijonas puede encontrarse en Urbina Rangel (1997). 
CHAUMEIL, J.-P., 2006 - Los orejones o gente-piraña. Percepción de la diferencia cultural en la Amazonia noroccidental. Anales del Museo Nacional de Antropología, XII: 51-62.

CONSEJO DE ANCIANOS DE LA ETNIA MUINANE, 1996 - Feene Jatyímeje Jatyímega 'Asiento azul de la tierra del centro': Memorias del territorio de la etnia muinane, 161 рр.; Putumayo, Amazonas, Colombia: Resguardo Indígena Predio. Manuscrito inédito. Editado por J. A. Echeverri, Fundación Gaia-Amazonas.

CORREDOR, B., 1986 - La maloca murui-muinane, 555 pp.; Bogotá: Universidad Nacional de Colombia, Departamento de Antropología. Tesis de Doctorado.

FRANKY CALVO, C. E., ZÁRATE, C. G. \& FRANCO, F., 2001 - Imani mundo. Estudios en la Amazonia colombiana, 558 pp.; Bogotá: Unibiblos.

GASCHÉ, J., 1976 - Les Fondements de l'organisation sociale des indiens Witoto et l'illusion exogamique. In: Actes du 42ème Congrès International des Américanistes, vol. 2: 141-161; París.

GASCHÉ, J., 2007 - Cuatro cantos-adivinanzas huitoto - Sociedad Bosquesina. Folia Amazónica, 16 (1-2): 89-100.

GOULARD, J.-P., 2011 - El nor-oeste amazónico en 1776: expediente sobre cumplimiento de la real cédula dada en San Ildefonson a 2 de septiembre de 1772, Vol. 1, xxvi + 173 pp; Leticia: Universidad Nacional de Colombia, Sede Amazonia.

GRIFFITHS, T. F. W., 1998 - Ethnoeconomics and native Amazonian livelihood: culture and economy among the Nipóde-Uitoto of the Middle Caquetá Basin in Colombia, 379 pp.; Oxford: University of Oxford. Tesis de doctorado.

GUYOT, M., 1972 - La maison des indiens Bora et Miraña. Journal de la Société des Américanistes, 61 (1): 141-176.

HUGH-JONES, S., 1996 - Bonnes raisons ou mauvaise conscience ? De I'ambivalence de certains Amazoniens envers la consommation de viande. Terrain, 26: 123-148.

HUGH-JONES, S., 2011 - La Palma y las Pléyades - Iniciación y cosmología en la Amazonia Noroccidental, 402 pp.; Bogotá: Universidad Central.

KARADIMAS, D., 2004 - La raison du corps : idéologie du corps et représentations de I'environnement chez les Mirana d'Amazonie colombienne, 451 pp.; París-Dundley: Peeters-Selaf. Coll. «Langues et sociétés d'Amérique traditionnelle »n. ${ }^{\circ} 9$.

KARADIMAS, D., 2005 - Chercher le centre : stratégie d'orientation spatiale chez les Miraña d'Amazonie colombienne. In: Les Espaces de l'homme (A. Berthoz \& R. Recht, eds.): 67-92; París: O. Jacob.

KUYOTEKA, A. \& RESTREPO, D., 1997 - Mitología Uitota: contada por un Aron +, «Gente de Avispa», 516 pp.; Medellín: Editorial Lealon.

LEACH et al., 1980 - L'unité de l'homme et autres essais, 396 pp.; París: Gallimard.

LONDOÑO SULKIN, C. D., 2004 - Muinane: un proyecto moral a perpetuidad, 339 pp.; Medellín: Editorial Universidad de Antioquia (Colección Antropología).

PINEDA CAMACHO, R., 2005 - Bajo el imperio del antropófago: las casas caníbales y los sacrificios humanos entre los uitotos de la Amazonía Colombiana. In: Chamanismo y sacrificio: perspectivas arqueológicas y etnológicas en sociedades indígenas de América del Sur (J.-P. Chaumeil, R. Pineda Camacho \& J.-F. Bouchard, eds.): 225 236; Bogotá: Banco de la República-Fundación de Investigaciones Arqueológicas Nacionales, Instituto Francés de Estudios Andinos.

PREUSS, K.T., 1994 - Religión y mitología de los Uitotos: recopilación de textos y observaciones efectuadas en una tribu indígena de Colombia, Suramérica, 918 pp.; Bogotá: Instituto Colombiano de Cultura, COA, Universidad nacional de Colombia. 
ROMAN, O.-E., ECHEVERRI, J. A. \& ROMAN, S., 2010 - Mito de la coca: el manejo y sus consecuencias. Mundo Amazónico, 1 (1): 315-326.

SEVERI, C., 1993 - La memoria rituale: follia e immagine del Bianco in una tradizione sciamanica amerindiana, 273 pp.; Firenze: Nuova Italia.

SEVERI, C., 2002 - Memory, reflexivity and belief. Reflections on the ritual use of language. Social Anthropology, 10 (1): 23-40.

SEVERI, C., 2007 - Le principe de la chimère : une anthropologie de la mémoire ?, 370 pp.; París: Éditions Rue d'Ulm-Ens, Musée du quai Branly.

TETEYE, R. \& TETEYE, E., 2005 - Nivel de desarrollo de la comunidad indígena de La Chorrera; Medellin: Universidad Pontificia Bolivariana. Tesis de Magister en participación y desarrollo comunitario.

URBINA RANGEL, F., 1997 - Un rito para hacer la paz. ¿Por qué los uitotos hacen baile de karijona? In: Religión y Etnicidad en América Latina, vol. 2 (G. Ferro, ed.): 79-127; Santafé de Bogotá: Instituto Colombiano de Antropología.

URBINA RANGEL, F., 2010 - Las palabras del origen: breve compendio de la mitología de los uitotos, 260 pp.; Bogotá: Ministerio de Cultura.

VIVEIROS DE CASTRO, E., 2002 - A inconstância da alma selvagem e outros ensaios de antropologia, 552 pp.; São Paulo: Cosac \& Naify. 